Ulf Andreasson*, Julia Kuhlmann, Josef Pannee, Robert M. Umek, Erik Stoops, Hugo Vanderstichele, Anja Matzen, Manu Vandijck, Martine Dauwe, Andreas Leinenbach, Sandra Rutz, Erik Portelius, Ingrid Zegers, Henrik Zetterberg and Kaj Blennow

\title{
Commutability of the certified reference materials for the standardization of $\beta$-amyloid 1-42 assay in human cerebrospinal fluid: lessons for tau and $\beta$-amyloid 1-40 measurements
}

https://doi.org/10.1515/cclm-2018-0147

Received February 7, 2018; accepted May 29, 2018; previously published online June 27, 2018

\section{Abstract}

Background: The core Alzheimer's disease cerebrospinal fluid (CSF) biomarkers total tau (T-tau), phosphorylated tau (P-tau), $\beta$-amyloid 1-42 (A $\beta 42)$ and $\beta$-amyloid 1-40 $(\mathrm{A} \beta 40)$ are increasing in importance and are now part of the research criteria for the diagnosis of the disease. The main aim of this study is to evaluate whether a set of certified reference materials (CRMs) are commutable for $\mathrm{A} \beta 42$ and to serve as a feasibility study for the other markers. This property is a prerequisite for the establishment of CRMs which will then be used by manufacturers to calibrate their assays against. Once the preanalytical factors have been standardized and proper selection criteria are available for subject cohorts this harmonization between methods will allow for universal cut-offs to be determined.

Methods: Thirty-four individual CSF samples and three different CRMs where analyzed for T-tau, P-tau, A $\beta 42$ and $A \beta 40$, using up to seven different commercially available methods. For $A \beta 40$ and $A \beta 42$ a mass spectrometry-based procedure was also employed.

\footnotetext{
*Corresponding author: Ulf Andreasson, PhD, Institute of Neuroscience and Physiology, Department of Psychiatry and Neurochemistry, The Sahlgrenska Academy at University of Gothenburg, 431 80, Mölndal, Sweden, Phone: +46 31 3430307, E-mail: ulf.andreasson@neuro.gu.se; and Clinical Neurochemistry Laboratory, Sahlgrenska University Hospital, Mölndal, Sweden

Julia Kuhlmann and Ingrid Zegers: European Commission, Joint Research Centre (JRC), Directorate F - Health, Consumers and Reference Materials, Geel, Belgium Josef Pannee, Erik Portelius and Kaj Blennow: Institute of Neuroscience and Physiology, Department of Psychiatry and Neurochemistry, The Sahlgrenska Academy at University of Gothenburg, Mölndal, Sweden; and Clinical Neurochemistry Laboratory, Sahlgrenska University Hospital, Mölndal, Sweden
}

Results: There were strong pairwise correlations between the different methods (Spearman's $\rho>0.92$ ) for all investigated analytes and the CRMs were not distinguishable from the individual samples.

Conclusions: This study shows that the CRMs are commutable for the different assays for $A \beta 42$. For the other analytes the results show that it would be feasible to also produce CRMs for these. However, additional studies are needed as the concentration interval for the CRMs were selected based on $\mathrm{A} \beta 42$ concentrations only and did in general not cover satisfactory large concentration intervals for the other analytes.

Keywords: Alzheimer's disease; biomarkers; commutability.

\section{Introduction}

Biomarkers are valuable tools that can be used in the clinical diagnostic process and as an inclusion criterion for clinical trials with the aim of finding disease modifying drugs [1]. In the field of Alzheimer's disease (AD) there are presently four core cerebrospinal fluid (CSF) biomarkers described in the literature with a potential clinical value: the 40 and 42 amino acid-long amyloid- $\beta$ (A 340 and $A \beta 42)$,

Robert M. Umek: Meso Scale Diagnostics, LLC, Rockville, MD, USA Erik Stoops and Hugo Vanderstichele: ADx NeuroSciences, Gent, Belgium

Anja Matzen: IBL International GmbH, Hamburg, Germany Manu Vandijck and Martine Dauwe: Fujirebio Europe N.V., Gent, Belgium

Andreas Leinenbach and Sandra Rutz: Roche Diagnostics GmbH, Penzberg, Germany

Henrik Zetterberg: Institute of Neuroscience and Physiology, Department of Psychiatry and Neurochemistry, The Sahlgrenska Academy at University of Gothenburg, Mölndal, Sweden; Clinical Neurochemistry Laboratory, Sahlgrenska University Hospital, Mölndal, Sweden; Department of Molecular Neuroscience, UCL Institute of Neurology, Queen Square, London, UK; and UK Dementia Research Institute, London, UK 
the microtubule-associated protein tau (T-tau), and its hyper phosphorylated variant (P-tau) [2]. In a large metaanalysis study, the concentrations of these core biomarkers in CSF were shown to be consistently altered in patients with $\mathrm{AD}$ compared to cognitively unimpaired individuals of a similar age [3]. Today these core biomarkers are only included in the research criteria for $\mathrm{AD}[4,5]$, but ideally uniform global cut-off levels will be established to facilitate a more general use, also in routine clinical diagnostics. However, this is partially hindered by the lack of harmonization between different methods and laboratories. Starting in 2009, efforts have been made to investigate this discord in detail when the Alzheimer's Association quality control (QC) program $[6,7]$ for CSF biomarkers was launched (www. Neurochem.gu.se/TheAlzAssQCProgram). A QC program by itself can do little to improve the situation but is a valuable tool in assessing the progress of method optimizations and to compare the quality in terms of variability and concentration differences between different assays. It may also increase the awareness of problems and engage laboratories that produce erroneous concentrations to revise their internal procedures for sample analysis. Partly driven by the rather discouraging results from the first rounds of the QC program [6, 7], manufacturers have worked on improving their assays and new fully automated methods with superior precision are emerging [8].

The lack of harmonization between different assays can be overcome by standardization through the development of reference measurement procedures (RMPs), the use of certified reference materials (CRMs) and the establishment of a traceability chain. The concept to use CSF for assay harmonization was described previously for the xMAP - enzyme-linked immunosorbent assay technology comparison, in which CSF A 342 concentrations were compared between assays using a CSF-calibrator series [9]. Therefore, the International Federation for Clinical Chemistry and Laboratory Medicine set up a working group with the aim to develop CRMs for Alzheimer's biomarkers in CSF
[10]. As a part of this process there is a need to show that reference materials are commutable for the different assays. The Vocabulary of Metrology [11] defines commutability as the "property of a reference material, demonstrated by the closeness of agreement between the relation among the measurement results for a stated quantity in this material, obtained according to two given measurement procedures, and the relation obtained among the measurement results for other specified materials". In other words, results obtained using different assays must show a high degree of correlation, and results from CRMs must be indistinguishable from representative individual samples for which the methods are intended to be used. Based on the results from a previous commutability study for $\mathrm{A} \beta 42$, it was obvious that artificial matrices (e.g. phosphate buffered saline, or artificial CSF), to which synthetic $A \beta 42$ was spiked, were not commutable [12]. As a consequence, CRMs were prepared from CSF pools with different endogenous concentrations of $A \beta 42$. The first aim for the current study was to examine if the CRMs are commutable with respect to $A \beta 42$. Samples of the three CRMs and 34 individual CSF samples were sent to five immunoassay manufacturers with commercially available assays for A 442 . In addition, commutability data for the other core biomarkers, i.e. T-tau and P-tau and $\mathrm{A} \beta 40$ (the two amino acid shorter and less aggregationprone $A \beta$ form that may be used to normalize individuals in regards to their constitutive $A \beta$ production rate [13]), were generated on the same sample set. The latter can be considered as a feasibility study for the development of CRMs for these other CSF biomarkers.

\section{Materials and methods}

\section{Samples}

The following CRMs were prepared, at three concentration levels, at the Joint Research Centre Directorate F - Health, Consumer and

Table 1: Summary of the sources for the data collected and their catalogue number.

\begin{tabular}{llllll}
\hline Manufacturer & Platform/principle & Aß42 & A 40 & T-tau & P-tau \\
\hline Sahlgren's University Hospital & LC-MS & In-house & In-house & - & - \\
Roche & Cobas & $06986811-190$ & - & Not released & Not released \\
IBL & ELISA & RE59661 & RE59651 & RE59631 & - \\
Euroimmun & ELISA & EQ-6521-9601-L & EQ-6511-9601-L & EQ-6531-9601-L & - \\
Meso Scale Diagnostics & ECL & K150SKE & K150SKE & K151LAG & - \\
Fujirebio & ELISA & 81576 & 81585 & 81572 & 81574 \\
Fujirebio & Modified ELISA & Not released & - & - & - \\
Fujirebio & Lumipulse & 230336 & - & 230312 & - \\
\hline
\end{tabular}

LC-MS, liquid chromatography-tandem mass spectrometry; ELISA, enzyme-linked immunosorbent assay; ECL, electrochemiluminescence. 

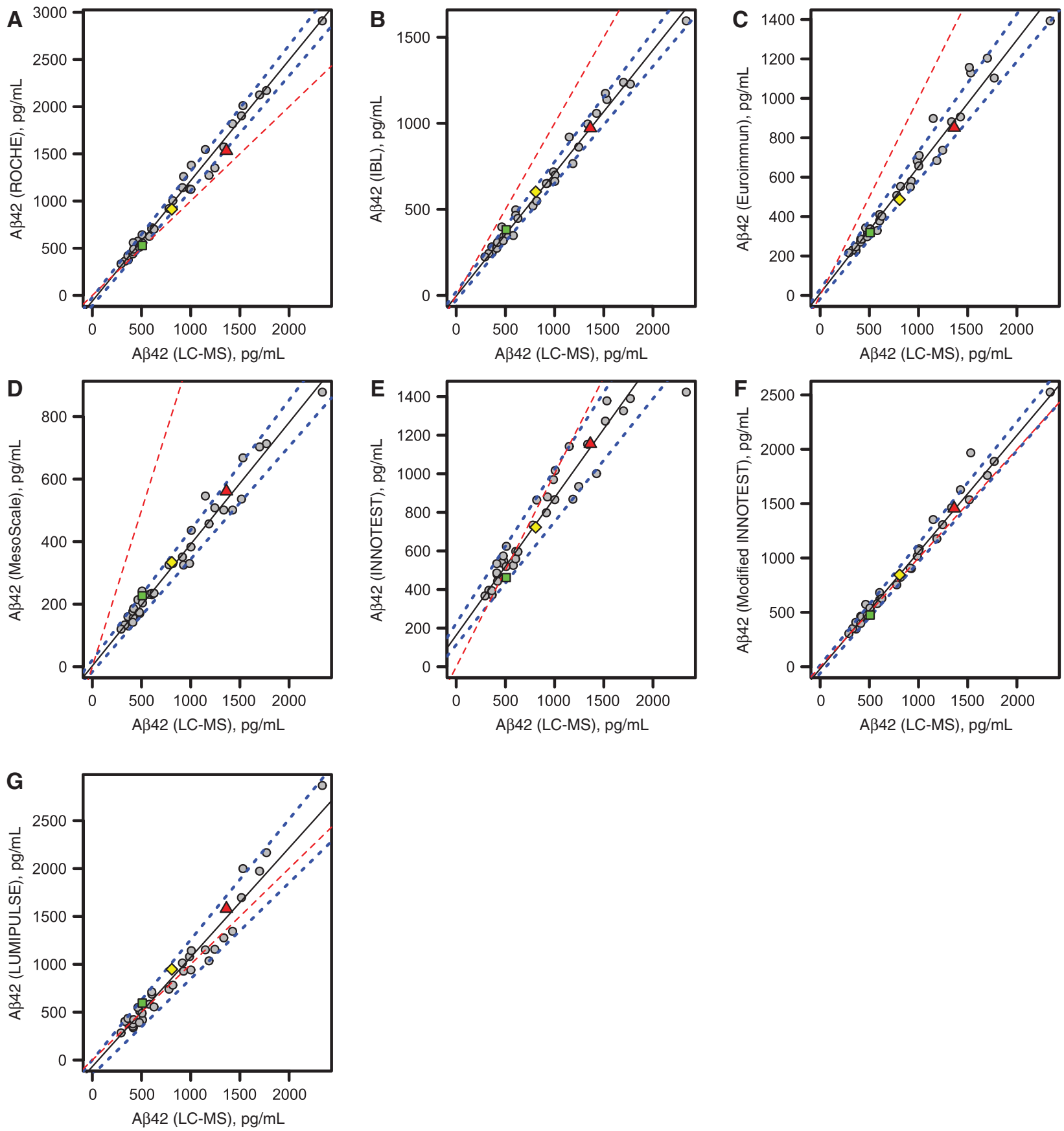

Figure 1: Scatter plots for pair-wise comparisons for A 342 with the LC-MS method against (A) ROCHE, (B) IBL, (C) Euroimmun, (D) MesoScale, (E) INNOTEST, (F) Modified INNOTEST and (G) LUMIPULSE.

The solid black line represents the Passing-Bablok regression and the upper blue dotted lines are constructed from the upper limit for the $95 \%$ confidence interval of the slope and intercept while the lower are based on the lower limits. The red dashed line denotes the unity line $(y=x)$. Symbols: individual CSF samples (gray circles); candidate CRMs (ERM-DA480/IFCC [green square], ERM-DA481/IFCC [yellow diamond], and ERM-DA482/IFCC [red triangle]).

Reference Materials: low (ERM-DA480/IFCC), middle (ERM-DA481/ IFCC) and high (ERM-DA482/IFCC). During the time period of this study, these CRMs have also been value assigned, and are available for use, see the Certification Report [14]. The target concentrations of the individual CSF samples were determined in advance using the
INNOTEST $\beta$-Amyloid (1-42). Aliquots of 34 individual CSF samples were selected at the Clinical Neurochemistry laboratory in Mölndal, Sweden, to cover the clinically relevant range of A 42 concentrations. Each individual CSF sample was divided into seven $500 \mu \mathrm{L}$ aliquots and in order to mitigate a potential bias from the order in which an 

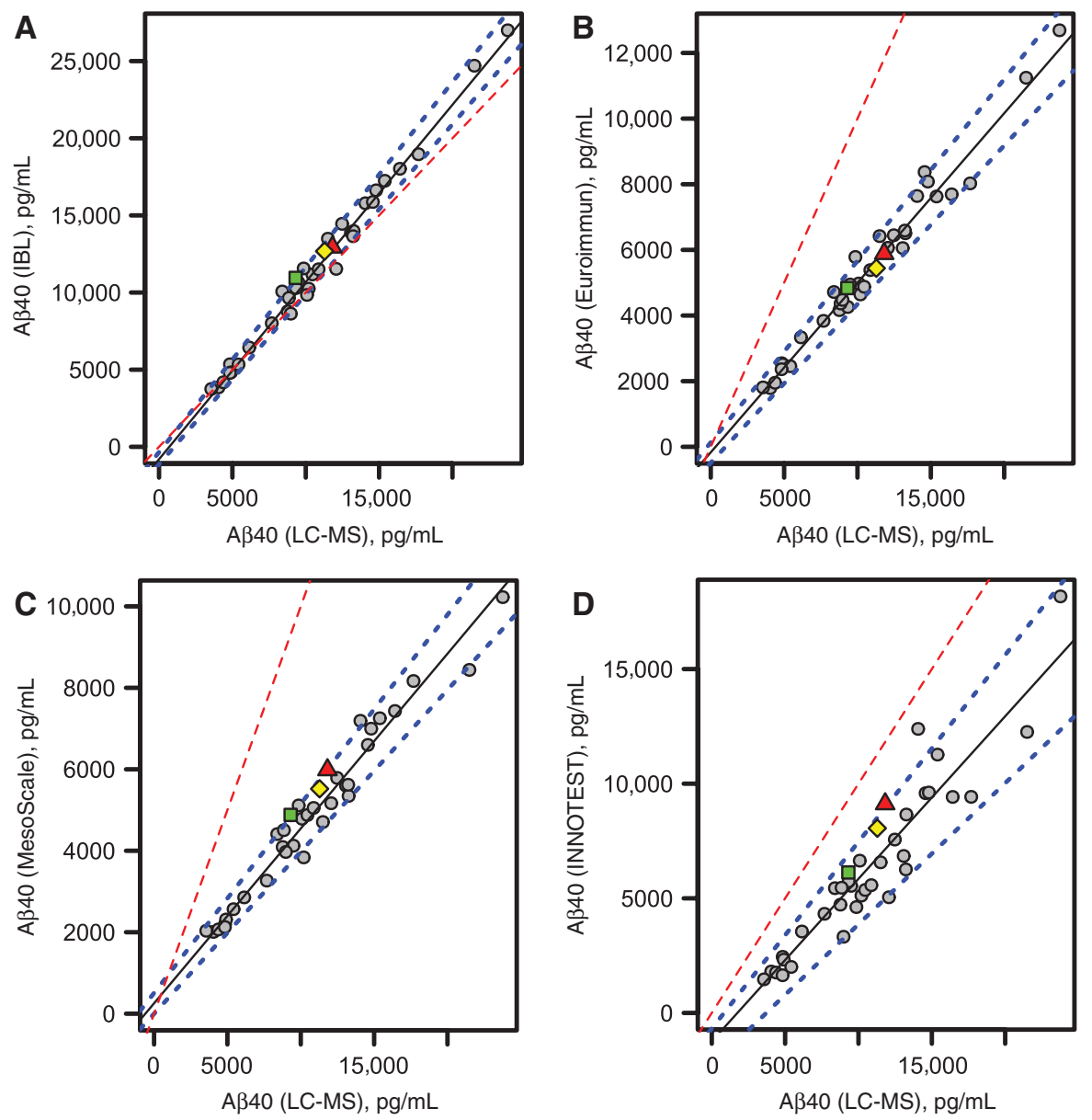

Figure 2: Scatter plots for pair-wise comparisons for A 340 with the LC-MS method against (A) IBL, (B) Euroimmun, (C) MesoScale and (D) INNOTEST.

The solid black line represents the Passing-Bablok regression and the upper blue dotted lines are constructed from the upper limit for the $95 \%$ confidence interval of the slope and intercept while the lower are based on the lower limits. The red dashed line denotes the unity line $(\mathrm{y}=\mathrm{x})$. Symbols: individual CSF samples (gray circles); candidate CRMs (ERM-DA480/IFCC [green square], ERM-DA481/IFCC [yellow diamond], and ERM-DA482/IFCC [red triangle]).

aliquot was prepared, each manufacturer received samples from different positions in the dispensing order. The same type of tube (Axygen; Product \#: SCT-050-SS-R) was used for both the CRMs and the individual samples. All samples were stored at $-80^{\circ} \mathrm{C}$ before shipment on dry ice to the different assay manufacturers for analysis. After the analysis the individual samples had undergone two freeze-thaw cycles.

\section{Assays}

The samples were sent to and analyzed by the manufacturers of the immunoassays (i.e. Euroimmun [analyzed by ADx in Gent], Fujirebio, IBL, Meso Scale Diagnostics and Roche) according to the instructions accompanying the kits. In addition a modified version of the INNOTEST $\beta$-Amyloid (1-42) assay (Fujirebio) was also evaluated [15]. The neurochemistry laboratory in Mölndal, Sweden performed measurements of $A \beta 42$ and $A \beta 40$ using liquid chromatographytandem mass spectrometry (LC-MS) [16]. Unless otherwise stated, all individual CSF samples were analyzed in duplicates while the CRMs were run in quadruplicates. Even though the main focus in this study was on $A \beta 42$ the immunoassay manufacturers were asked to measure the other biomarkers using the priority list $\mathrm{A} \beta 40, \mathrm{~T}$-tau and P-tau, depending on sample volume left after $\mathrm{A} \beta 42$ analysis and availability of methods on their platforms (Table 1).

\section{Statistics}

Statistical analyses were conducted in the statistical environment R 3.4.0 [17]. Commutability was evaluated using Passing-Bablok regression [18] (package 'mcr' [19]) and Spearman's rank correlation coefficient $\left(\mathrm{r}_{\mathrm{s}}\right)$. The normalized distance to the regression line, in unit per cent, for the samples was calculated as the Euclidean norm. To determine if the CRMs are comparable to individual samples the effect size, based on the distances to the regression line for the two groups, was estimated with the area under the receiver operating characteristic curve (AUROC) and the 95\% confidence interval (95\% CI) (package 'pROC' [20]). If the $95 \%$ CI contains 0.5 there is 

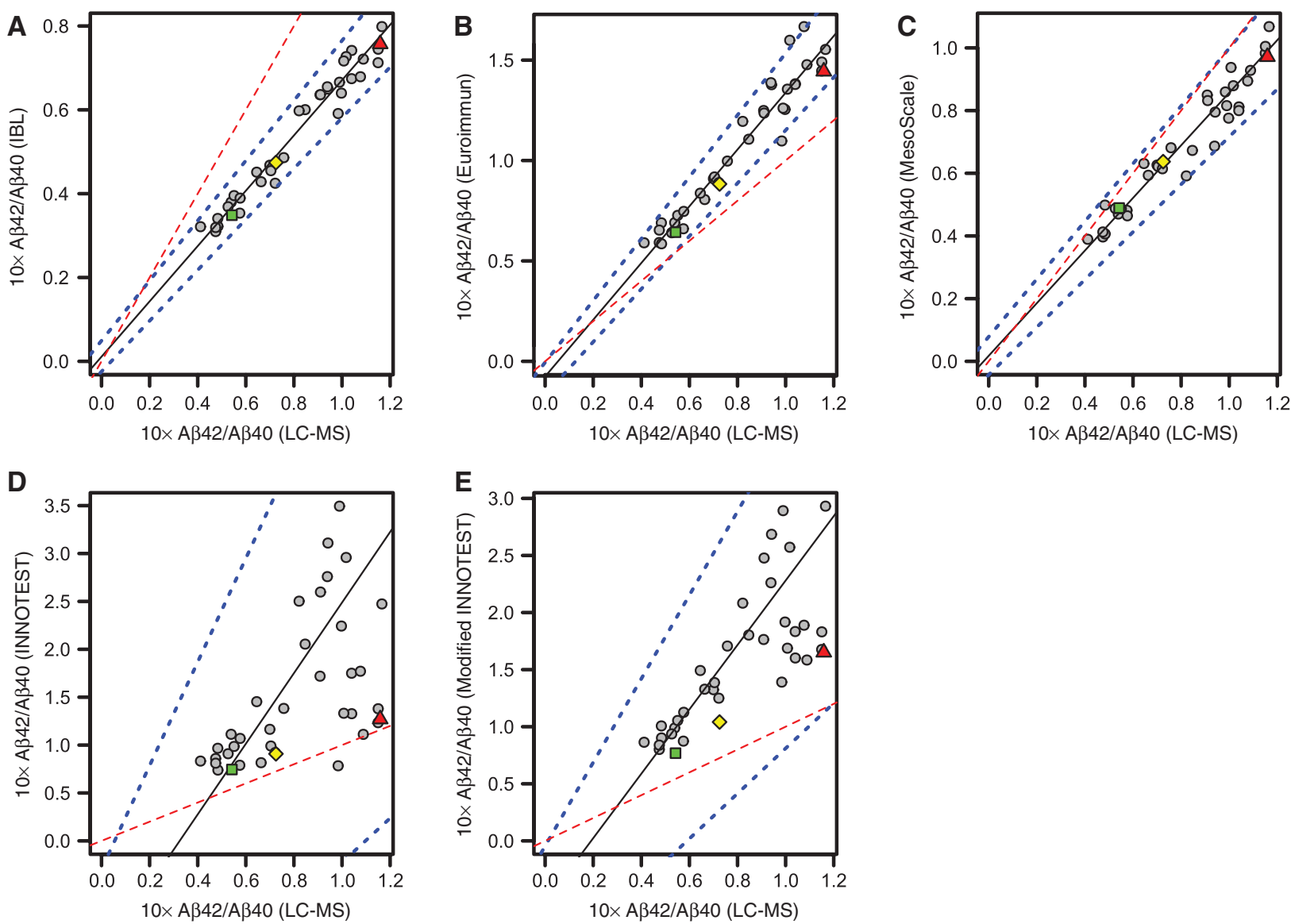

Figure 3: Scatter plots for pair-wise comparisons for 10xA $342 / A \beta 40$ with the LC-MS method against (A) IBL, (B) Euroimmun, (C) MesoScale, (D) INNOTEST and (E) Modified INNOTEST.

The solid black line represents the Passing-Bablok regression and the upper blue dotted lines are constructed from the upper limit for the $95 \%$ confidence interval of the slope and intercept while the lower are based on the lower limits. The red dashed line denotes the unity line $(\mathrm{y}=\mathrm{x})$. Symbols: individual CSF samples (gray circles); candidate CRMs (ERM-DA480/IFCC [green square], ERM-DA481/IFCC [yellow diamond] and ERM-DA482/IFCC [red triangle]).

no statistical difference between the groups. Linearity of the associations was tested using the F-test for goodness of fit between two assays comparing the nested linear and second order polynomial functions.

\section{Results}

The different assays that were used in this study are listed in Table 1. For the assay from IBL one result for T-tau was not reported and this sample was excluded in the subsequent analysis for all other T-tau assays as well. For T-tau measured on the Lumipulse platform only one result per sample was reported due to shortage of sample volume. Over all the repeatability, based on duplicate determinations, was very good for all assays and analytes with an average coefficient of variation per method of $0.4 \%-5.9 \%$.
The correlations, using data only from individual samples, for all pair-wise method comparisons for A 342 were strong with a median correlation coefficient of 0.98 (min-max: 0.94-0.99) (Figure 1, Supplementary Figure 1 and Supplementary Table 1). The slopes of the regression lines were in the interval 0.31-2.99 (Supplementary Table 1) and the normalized distance to the regression line for the individual samples and the CRMs were not statistically significant at the $95 \%$ confidence level (Supplementary Figure 2A) with an AUROC of 0.557 (95\% CI: 0.495-0.619).

For $A \beta 40$ measurements the correlations were strong as well with a median correlation coefficient of 0.97 (min-max: 0.93-0.98) (Figure 2, Supplementary Figure 3 and Supplementary Table 2). The distance to the regression line for the individual samples and the CRMs were also not statistically significant at the 95\% confidence level (Supplementary Figure 2B) with an AUROC of 0.598 (95\% CI: 0.496-0.701). 
The distribution range of the CRMs was smaller for $A \beta 40$ as compared to $A \beta 42$, attributed to the fact that only the $A \beta 42$ concentrations were considered when preparing CSF pools used for the production of the CRMs. Furthermore, the individual samples were selected solely based on the clinically relevant range for $\mathrm{A} \beta 42$ but nevertheless these samples still cover an adequate range for $\mathrm{A} \beta 40$.

Even though the correlation was strong between different assays for both $A \beta 42$ and $A \beta 40$ this did not generally hold true for the $A \beta 42 / A \beta 40$ ratio (Figure 3, Supplementary Figure 4 and Supplementary Table 3). The correlations had a median of 0.85 (min-max: 0.58-0.97). Comparing the correlations for the components of the ratio, the main contribution for the lower correlations seemed to come from $\mathrm{A} \beta 40$ and the variability increased for the ratio as a consequence of the propagation of uncertainty. The CRMs essentially covered the range defined by the individual samples and it can be noted that in all method comparisons for the ratio, the three CRMs fell closely on a straight line even when the variability for the individual samples was high (Figure 3 and Supplementary Figure 4). The effect size indicated that there was no statistical difference between the individual samples and the CRMs at the 95\% confidence level with an AUROC of 0.576 (95\% CI: 0.481-0.671; Supplementary Figure 2C).

For T-tau the correlations were strong for all assays used (Supplementary Figure 5 and Supplementary Table 4) with a median of 0.97 (min-max: 0.92-0.99). Although there was a small difference between the CRMs, with regard to the measured concentration, none of them deviate substantially from the regression line (Supplementary Figure 5) and they behave very much like the individual samples (Supplementary Figure 2D) with an AUROC of 0.564 (95\% CI: 0.471-0.656). The same is true for P-tau for the only pair of assays that were included in the study, where the correlation was $r_{s}=0.975$ and the slope of the regression line was 2.55 (Supplementary Figure 6). As there were only three results for the CRMs (Supplementary Figure 2E) the uncertainty of the effect size was large with an AUROC of 0.657 (95\% CI: 0.392-0.922).

The F-test for goodness of fit comparing the nested linear or second order polynomial fit revealed that both INNOTEST and Lumipulse had a $\mathrm{p}<0.05$ for all pairwise comparisons of $\mathrm{A} \beta 42$ measurements. This indicates that the associations were non-linear. The F-test results were not statistically significant at the $95 \%$ confidence level for any of the other $A \beta 42$ method comparisons. For Aß40 measurements, two out of four comparisons with MSD (i.e. vs. INNOTEST and IBL) indicated non-linearity. Of the ten possible combinations for T-tau assays, three had a $\mathrm{p}<0.05$ for the F-test (Roche vs. Euroimmun, Euroimmun vs. INNOTEST, and INNOTEST vs. MSD).

\section{Discussion}

The presented results demonstrate that the approach to prepare CRMs for $\mathrm{A} \beta 42$ from pooled CSF samples can be considered valid. All three CRMs are commutable for all combinations of methods and all immunoassay methods for A 42 show a strong correlation of results with the results obtained with the LC-MS method. This is a prerequisite for the use of the RMP, which is also a LC-MS method, for the value-assignment of the CRMs.

One of the potential confounding factors for the LC-MS method is the methionine residue on position 35 in the $A \beta 42$ sequence which can be subjected to oxidation forming a methionine sulfoxide [21]. This type of post translational modification has a biological function in other proteins [22] and it can also form in vitro on $\mathrm{A} \beta 42$ [23]. The LC-MS only measures non-oxidized A $\beta 42$, as opposed to the antibody-based methods that are expected to not discriminate between the two forms. However, strong correlations between LC-MS and the other methods suggest that the fraction of oxidized $A \beta 42$ is very low and/or constant. Thus, oxidation of methionine in $\mathrm{A} \beta 42$ is insignificant in this system. The same reasoning and conclusion holds true also for $A \beta 40$.

By itself, CSF $A \beta 40$ is not a biomarker with a high clinical utility in the field. However, the ratio of $A \beta 42 /$ $A \beta 40$ seems to improve the diagnostic performance [24, 25] and it also shows better concordance with amyloid positron emission tomography $[16,26]$, which can be considered the gold standard for imaging the $A \beta$ pathology in the brain. A candidate RMP for $A \beta 40$ has been submitted to Joint Committee for Traceability in Laboratory Medicine for approval as there is a need for a CRM for this marker as well to establish a global cutoff of the $A \beta 42 / A \beta 40$ ratio. However, the three CRMs for $\mathrm{A} \beta 42$ do not cover an appropriate clinical range of $A \beta 40$ concentrations, which means that separate CRMs for $A \beta 40$ are needed. Nevertheless, the presented results serve as a successful feasibility study for developing CRMs for $\mathrm{A} \beta 40$. The feasibility also applies to T-tau and P-tau, even though the latter only involved two different methods in this study. Also for T-tau and P-tau, the CRMs investigated do not span the clinically relevant range of concentrations and therefore new CRMs will have to be prepared to verify the concept of using the same type of materials. The protocol to prepare CSF and impact of confounding factors during storage and collection is much more robust for tau, P-Tau and other proteins as compared to A $\beta 1-42$ [27]. Work is ongoing to establish a LC-MS method for T-tau, which is within reach, while the sensitivity needed for P-tau makes a MS based method for this analyte a bigger challenge. Compared to 
A $\beta 42$ the other analytes showed less systematic deviation from linearity for any of the assays.

Non-linear association between different methods might pose a problem for the harmonization of global diagnostic cutoffs and manufacturers should take measures to ensure that their methods have linear associations with the RMP. It can be that the association is in fact linear and that a few erroneous results drive the non-linear appearance highlighting the need of replication studies. While this might be true, for example, for A $\beta 42$ measurements on Lumipulse, the INNOTEST assay appears to be non-linear when compared to other methods. This has already been noticed in a previous commutability study although the linearity issue was not assessed in detail [12]. In this regard the modified INNOTEST [15] is an improvement as the test did not indicate non-linearity for any comparisons. It is important to keep in mind that the concentration of the calibrator used with the LC-MS is not fully characterized, and deviations from the unity line might be attributed to the uncertainty of the concentration of the calibrator used.

Harmonization of methods used to measure biomarkers is important for the establishment of global cutoff levels but pre-analytical confounding factors also contribute to the variability of the measured concentrations of the biomarkers [28-33]. One aim with the BIOMARKAPD project in the framework of the Joint Programming Neurodegenerative Disease was to address this source of error resulting in recommendations for pre-analytical handling of CSF samples [34]. Within the framework for the Alzheimer's Association QC program for CSF biomarkers an attempt was made to find methodological factors that contributed to the variability in the reported results. A questionnaire was sent out in which the participants were asked to answer questions about instrumentation, equipment, training of staff, storage of samples and kits, etc. From the replies, no conclusion could be made regarding specific factors that potentially contributed more to the variability than others (unpublished results). The human factor is possibly a great contributor to the variability and fully automated assays such as the Cobas (Roche) and the Lumipulse (Fujirebio) systems, included in this commutability study, have the potential to minimize human interference on the results. Other random-access analyzers will be available in the future, such as qualified by Euroimmun [30]. Although these platforms are not new to the market they are not commonly used for AD biomarkers. It remains to be seen if they surpass the more manual methods with regard to precision in the future. Results from the initial rounds in the QC program in which these platforms were included indicate that this might be the case (www.Neurochem.gu.se/TheAlzAssQCProgram).
The materials both in the external QC program and in the CRMs are pooled leftover CSF samples which mimic patient samples well, as show in this study. This property is requested for internal QC samples according to ISO 15189 but the CRMs are primarily to be used by kit manufacturers and not by individual medical laboratories. If pooled CSF is not available in practice, internal artificial QC samples can be prepared that fulfill the criterion of similarity with patient samples [35]. However, not all artificial matrices commute [12] and this must be taken into account before an internal QC program is established.

In summary, the results presented supported the work for releasing the first CRMs for CSF measurements of $A \beta 42$. For the other biomarkers (i.e. A $\beta 40, \mathrm{~T}$-tau, and P-tau) more work is needed, but the present results of the feasibility are encouraging. The value assignment of the CRMs is completed and the CRMs have been released and available for purchase from the Joint Research Centre (crm.jrc.ec.europa. eu). They can now be used by the immunoassay manufacturers to recalibrate their assays as mandated in the EU Directive on In Vitro Diagnostics Medical Devices (Directive 98/79/EC). The release of the materials should improve the concordance of measurement results between different analytical platforms as well as laboratories and will allow the establishment of a global diagnostic cutoff value for A 342 in CSF that can be used for the diagnosis of AD. The arrival of a global diagnostic cutoff is positive but the pedagogical hurdle of informing the physicians, and make them accept the new reality, should not be neglected.

Author contributions: All the authors have accepted responsibility for the entire content of this submitted manuscript and approved submission.

Research funding: None declared. Employment or leadership: None declared.

Honorarium: None declared.

Competing interests: The funding organization(s) played no role in the study design; in the collection, analysis, and interpretation of data; in the writing of the report; or in the decision to submit the report for publication.

\section{References}

1. Lewczuk P, Riederer P, O’Bryant SE, Verbeek MM, Dubois B, Visser PJ, et al. Cerebrospinal fluid and blood biomarkers for neurodegenerative dementias: an update of the Consensus of the Task Force on Biological Markers in Psychiatry of the World Federation of Societies of Biological Psychiatry. World J Biol Psychiatry 2018;19:244-328. 
2. Blennow K, Hampel H, Weiner M, Zetterberg H. Cerebrospinal fluid and plasma biomarkers in Alzheimer disease. Nat Rev Neurol 2010;6:131-44.

3. Olsson B, Lautner R, Andreasson U, Ohrfelt A, Portelius E, Bjerke $M$, et al. CSF and blood biomarkers for the diagnosis of Alzheimer's disease: a systematic review and meta-analysis. Lancet Neurol 2016;15:673-84.

4. Dubois B, Feldman HH, Jacova C, Hampel H, Molinuevo JL, Blennow K, et al. Advancing research diagnostic criteria for Alzheimer's disease: the IWG-2 criteria. Lancet Neurol 2014;13:614-29.

5. McKhann GM, Knopman DS, Chertkow H, Hyman BT, Jack CR Jr, Kawas $\mathrm{CH}$, et al. The diagnosis of dementia due to Alzheimer's disease: recommendations from the National Institute on AgingAlzheimer's Association workgroups on diagnostic guidelines for Alzheimer's disease. Alzheimers Dement 2011;7:263-9.

6. Mattsson N, Andreasson U, Persson S, Arai H, Batish SD, Bernardini S, et al. The Alzheimer's association external quality control program for cerebrospinal fluid biomarkers. Alzheimers Dement 2011;7:386-95.e6.

7. Mattsson N, Andreasson U, Persson S, Carrillo MC, Collins S, Chalbot S, et al. CSF biomarker variability in the Alzheimer's association quality control program. Alzheimers Dement 2013;9:251-61.

8. Bittner T, Zetterberg H, Teunissen CE, Ostlund RE Jr, Militello $\mathrm{M}$, Andreasson U, et al. Technical performance of a novel, fully automated electrochemiluminescence immunoassay for the quantitation of beta-amyloid (1-42) in human cerebrospinal fluid. Alzheimers Dement 2016;12:517-26.

9. Vanderstichele HM, Shaw L, Vandijck M, Jeromin A, Zetterberg $\mathrm{H}$, Blennow K, et al. Alzheimer disease biomarker testing in cerebrospinal fluid: a method to harmonize assay platforms in the absence of an absolute reference standard. Clin Chem 2013;59:710-2.

10. Kuhlmann J, Andreasson U, Pannee J, Bjerke M, Portelius E, Leinenbach A, et al. CSF Abeta1-42 - an excellent but complicated Alzheimer's biomarker - a route to standardisation. Clin Chim Acta 2016;467:27-33.

11. JCGM 200:2012 re. International vocabulary of metrology - basic and general concepts and associated terms. Paris: JCGM, 2012.

12. Bjerke M, Andreasson U, Kuhlmann J, Portelius E, Pannee J, Lewczuk $P$, et al. Assessing the commutability of reference material formats for the harmonization of amyloid beta measurements. Clin Chem Lab Med 2016;54:1177-91.

13. Lewczuk P, Matzen A, Blennow K, Parnetti L, Molinuevo JL, Eusebi P, et al. Cerebrospinal fluid Abeta42/40 corresponds better than Abeta42 to amyloid PET in Alzheimer's disease. J Alzheimers Dis 2017;55:813-22.

14. Kuhlmann J, Boulo S, Andreasson U, Bjerke M, Pannee J, Charoud-Got J, et al. CERTIFICATION REPORT: the certification of amyloid $\beta 1-42$ in CSF in ERM ${ }^{\circledR}$-DA480/IFCC, ERM ${ }^{\circledR}$-DA481/IFCC and ERM ${ }^{\circledR}$-DA482/IFCC: European Commission, 2017. Available at: https://ec.europa.eu/jrc/en/publication/eur-scientific-andtechnical-research-reports/certification-report-certificationamyloid-1-42-csf-erm-da480ifcc-erm-da481ifcc-and-erm.

15. Vandijck M, Moonen R, Andreasson U, Le Bastard N, Dauwe $\mathrm{M}$, Blennow K, et al. Correlation of the modified INNOTEST $\beta$-amyloid 1-42 with a LC-MS/MS candidate reference method. Alzheimers Dement 2015;11:P385.
16. Pannee J, Portelius E, Minthon L, Gobom J, Andreasson U, Zetterberg $\mathrm{H}$, et al. Reference measurement procedure for CSF amyloid beta (Abeta)1-42 and the CSF Abeta1-42/Abeta1-40 ratio - a cross-validation study against amyloid PET. J Neurochem 2016;139:651-8.

17. Team RC. R: a language and environment for statistical computing. Vienna, Austria: R Foundation for Statistical Computing, 2015. Available at: https://www.R-project.org/.

18. Passing H, Bablok W. A new biometrical procedure for testing the equality of measurements from two different analytical methods. Application of linear regression procedures for method comparison studies in clinical chemistry, Part I. J Clin Chem Clin Biochem 1983;21:709-20.

19. Manuilova E, Schuetzenmeister A, Model F. mcr: method comparison regression. $\mathrm{R}$ package version 1.2.1, 2014. Available at: https://CRAN.R-project.org/package=mcr.

20. Robin X, Turck N, Hainard A, Frédérique NT, Sanchez J, Müller M. pROC: an open-source package for R and $\mathrm{S}+$ to analyze and compare ROC curves. BMC Bioinformatics 2011;12:77.

21. Butterfield DA, Boyd-Kimball D. The critical role of methionine 35 in Alzheimer's amyloid beta-peptide (1-42)-induced oxidative stress and neurotoxicity. Biochim Biophys Acta 2005; 1703:149-56.

22. Vogt W. Oxidation of methionyl residues in proteins: tools, targets, and reversal. Free Radic Biol Med 1995;18:93-105.

23. Cohen SL. Ozone in ambient air as a source of adventitious oxidation. A mass spectrometric study. Anal Chem 2006;78:4352-62.

24. Lewczuk P, Esselmann H, Otto M, Maler JM, Henkel AW, Henkel MK, et al. Neurochemical diagnosis of Alzheimer's dementia by CSF Abeta42, Abeta42/Abeta40 ratio and total tau. Neurobiol Aging 2004;25:273-81.

25. Lewczuk P, Lelental N, Spitzer P, Maler JM, Kornhuber J. Amyloidbeta $42 / 40$ cerebrospinal fluid concentration ratio in the diagnostics of Alzheimer's disease: validation of two novel assays. J Alzheimers Dis 2015;43:183-91.

26. Janelidze S, Zetterberg H, Mattsson N, Palmqvist S, Vanderstichele $\mathrm{H}$, Lindberg 0 , et al. CSF Abeta42/Abeta40 and Abeta42/Abeta38 ratios: better diagnostic markers of Alzheimer disease. Ann Clin Transl Neurol 2016;3:154-65.

27. Vanderstichele H, Demeyer L, Janelidze S, Coart E, Stoops E, Mauroo K, et al. Recommendations for cerebrospinal fluid collection for the analysis by ELISA of neurogranin trunc P75, alpha-synuclein, and total tau in combination with Abeta(1-42)/ Abeta(1-40). Alzheimers Res Ther 2017;9:40.

28. Bjerke M, Portelius E, Minthon L, Wallin A, Anckarsater H, Anckarsater R, et al. Confounding factors influencing amyloid Beta concentration in cerebrospinal fluid. Int J Alzheimers Dis 2010;2010:1-11.

29. Perret-Liaudet A, Pelpel M, Tholance Y, Dumont B, Vanderstichele $\mathrm{H}$, Zorzi W, et al. Cerebrospinal fluid collection tubes: a critical issue for Alzheimer disease diagnosis. Clin Chem 2012;58:787-9.

30. Toombs J, Foiani MS, Paterson RW, Heslegrave A, Wray S, Schott JM, et al. Effect of spinal manometers on cerebrospinal fluid amyloid-beta concentration. J Alzheimers Dis 2017;56:885-91.

31. Toombs J, Paterson RW, Schott JM, Zetterberg H. Amyloid-beta 42 adsorption following serial tube transfer. Alzheimers Res Ther 2014;6:5. 
32. Toombs J, Paterson RW, Lunn MP, Nicholas JM, Fox NC, Chapman $M D$, et al. Identification of an important potential confound in CSF AD studies: aliquot volume. Clin Chem Lab Med 2013;51:2311-7. 33. Schoonenboom NS, Mulder C, Vanderstichele H, Van Elk EJ, Kok A, Van Kamp GJ, et al. Effects of processing and storage conditions on amyloid beta (1-42) and tau concentrations in cerebrospinal fluid: implications for use in clinical practice. Clin Chem 2005;51:189-95.

34. del Campo M, Mollenhauer B, Bertolotto A, Engelborghs S, Hampel H, Simonsen AH, et al. Recommendations to standardize preanalytical confounding factors in Alzheimer's and
Parkinson's disease cerebrospinal fluid biomarkers: an update. Biomark Med 2012;6:419-30.

35. Lelental N, Brandner S, Kofanova O, Blennow K, Zetterberg $\mathrm{H}$, Andreasson $\mathrm{U}$, et al. Comparison of different matrices as potential quality control samples for neurochemical dementia diagnostics. J Alzheimers Dis 2016;52:51-64.

Supplementary Material: The online version of this article offers supplementary material (https://doi.org/10.1515/cclm-2018-0147). 